\title{
Rheological Studies Dedicated to the Development of a Novel Injectable Polymeric Blend for Viscosupplementation Treatment
}

\author{
R. Lapasin, ${ }^{a}$ F. Segatti, ${ }^{b}$ D. Mercuri, ${ }^{b}$ G. De Conti, ${ }^{b}$ C. Spagnul, ${ }^{b}$ and S. Fusi ${ }^{\text {b }}$ \\ ${ }^{a}$ Department of Engineering and Architecture, University of Trieste, \\ via A. Valerio 6/A, I-34127 Trieste, Italy \\ ${ }^{\mathrm{b}} \mathrm{R} \& D$ Department, Limacorporate S.p.a., via Nazionale 52, \\ I-33038 Villanova di San Daniele del Friuli, Italy \\ doi: 10.15255/CABEQ.2014.2148 \\ Original scientific paper \\ Received: November 15, 2014 \\ Accepted: November 30, 2015
}

Viscosupplementation is an intra-articular symptomatic treatment of mild osteoarthritis. The treatment involves the injection of high-molecular-weight hyaluronan (HA), and especially of cross-linked HA to restore the lubricating and cushioning properties of the synovial fluid.

This work involves the development of a novel viscosupplementation fluid based on amidated carboxymethylcellulose and obtained by blending the soluble polymer with its crosslinked derivative. Rheological analyses carried out under both oscillatory and continuous shear provided a rationale to assess the viscosupplement formulation and the production process. The hydrogel fraction content and the total polymer concentration can be properly selected in order to ensure an optimal combination of flowability and viscoelastic properties.

Key words:

amidated carboxymethylcellulose, viscosupplementation, polymeric blend, hydrogel, rheology

\section{Introduction}

Osteoarthritis (OA) is a chronic, progressive musculoskeletal disorder ${ }^{1-3}$ that mainly affects the weight-bearing chondro-synovial joints such as knee, hip ${ }^{4}$ and the upper extremities. ${ }^{5,6}$ The disease affects the ageing population worldwide ${ }^{7,8}$ leading to a high socio-economic burden ${ }^{9}$ with substantial financial consequences. ${ }^{10,11}$

In a healthy condition, the lubricants present in the synovial fluid, such as Hyaluronic acid (HA), lubricin $^{12}$ and the surface-active phospholipids (SAPL) $)^{13,14}$ act as shock absorbers protecting the joint against compressive damage. ${ }^{15-17}$ Hyaluronic acid (HA) is responsible for the viscoelastic properties. $^{18}$

In OA joint diseases, the concentration and molecular weight of HA decrease ${ }^{16,19}$ and the synovial fluid becomes more abundant, less viscous and reduced in elastoviscous properties that may culminate in a painful condition for the patient.

Viscosupplementation ${ }^{20,21}$ was identified as an effective treatment of OA with a small number of adverse effects. Nowadays, it is approved in Europe/USA for the treatment of pain associated with OA of the knee, but considerable controversy about its efficacy is still ongoing as evidenced in the

"Corresponding author: romano.lapasin@di3.units.it
OARSI treatment guidelines. ${ }^{22}$ Viscosupplementation consists in intra-articular, single or repeated, injections of HA or HA-based derivatives (HAs). Adequate viscoelastic properties are also important to restore the articular homeostasis. ${ }^{23} \mathrm{HA}$ or HAs increase the elastoviscosity of the intercellular matrix creating a mechanical protective socket that surrounds nociceptive nerve endings. This results in a decrease in pain and an increase in joint mobility ${ }^{24}$ as it has already been demonstrated on animals models. ${ }^{25,26}$

One of the most important drawbacks of HA and HAs is the multiple injection therapy due to the enzymatic degradation by hyaluronidases in the body. High molecular weight products and crosslinked hydrogels have been developed, but they are able to retard the hyaluronidases attack without preventing it. ${ }^{27}$ Furthermore, inflammatory reactions sometimes accompanied by swelling and erythema have also been variably reported. ${ }^{28,29}$

A new derivative similar to HA, but with a better behaviour in terms of hardness and stability towards enzymatic degradation ${ }^{30}$ has been developed converting $50 \%$ of the carboxylic groups of carboxymethylcellulose (CMC) into amidic groups to obtain the amidated carboxymethylcellulose (CMCA). ${ }^{31}$ In vitro results showed that the amidation significantly ameliorated the biocompatibility of the material and diminished the cell expression 
of inflammatory cytokines. CMCA also positively influenced osteoblast activity. ${ }^{30}$ The vegetal origin of CMCA potentially avoids the risk of allergic reactions due to certain protein components present in the HA and Ha-based products injected.

This work involves the development of a novel viscosupplementation fluid obtained by blending the soluble CMCA polymer (hereinafter indicated as CMCAp) with its crosslinked hydrogel derivative (CMCAg) by crosslinking the residual $50 \%$ fraction of CMCAp carboxylic groups.

CMCA-based fluids were investigated to assess the rheological properties of the viscosupplement fluid during the different processing stages. Both continuous and oscillatory shear tests showed that, at equal polymer concentration, aqueous CMCAp and CMCAg systems exhibit quite different rheological behaviours, typical of polymer solutions and gels, respectively, and hence a wide arc of viscous and viscoelastic responses can be obtained by blending the two polymers in different proportions. The gel fraction (CMCAg/CMCAp ratio) and the total polymer concentration of the blend can be appropriately selected in order to ensure an optimal combination of viscoelastic properties and flow behaviour (injectability) together with an acceptable structural recovery after shear.

\section{Materials and methods}

\section{Materials}

The raw material for CMCA preparation is sodium carboxymethylcellulose (average molecular weight: $750 \mathrm{kDa}$, substitution degree 0.8 ) (CP Kelko). It can be functionalized thanks to the presence of two carboxyl groups in the polymeric unit through a water-based synthetic pathway. The carboxylic groups are first activated with NHS/EDC (N-hydroxysuccinimide purity $>97 \%$ (Sigma-Aldrich); N-(3-dimethylaminopropyl)-N'-ethylcarbodiimide hydrochloride purity $>99 \%$ (Actim)). Once activated, they quickly react with methylamine (40\% water solution, Sigma-Aldrich), molar ratio of $1: 1$. The $\mathrm{pH}$ of the solution is always kept at 4.5-5 adjusting hydrochloric acid. The amide-CMCA was then purified in a buffered solution (about $\mathrm{pH}$ 8-8.5 in order to increase the solubility of the polymer) through a Tangential Flow Filtration instrument using a membrane with $100 \mathrm{kDa}$ cut off. The obtained solution was separated into two aliquots: the former was freeze-dried, the latter was used for the crosslinking process.

The amide-CMC polymer is crosslinked by a solution of NHS/EDC and a bi-functional group. In particular, 1,3-diaminopropane (purity $99.0 \%$, Sigma-Aldrich) was used as crosslinking agent. The molar ratio with the polymer was $0.5: 1$ to obtain a crosslinking degree of about $50 \%$. The obtained hydrogel was purified by repeated rinsing in purified water and freeze dried. The two freeze-dried components, polymer and hydrogel, were milled in order to have a controlled cut off, up to $2 \mathrm{~mm}$, that were subsequently blended and hydrated in saline solution, and eventually filled in syringe and sterilized.

\section{Rheological experiments}

Rheological characterization was carried out with a rotational controlled stress rheometer (Rheostress RS150, Haake GmbH, Germany), equipped with a cone/plate device $(d=60 \mathrm{~mm}$, angle $\left.=1^{\circ}\right)$ or a parallel plate geometry $(d=35 \mathrm{~mm}$, gap $=1 \mathrm{~mm}$ ) with serrated surfaces in order to avoid wall slippage effects. The apparatus was computer-controlled (RheoWin software program) and the temperature was kept at $25 \pm 0.1{ }^{\circ} \mathrm{C}$ (Thermostat TC500, Haake GmbH, Germany). The flow properties were determined applying a logarithmic sequence of increasing constant stresses in the range 1-1000 $\mathrm{Pa}$ and measuring the corresponding shear rate. Each stress value was kept constant until the corresponding time variation of the shear rate satisfied the pre-set constraint for steady state attainment, or at most for $90 \mathrm{~s}$.

Oscillatory stress sweep tests were performed at $1 \mathrm{~Hz}$ in order to individuate the extension of the linear viscoelasticity regime. All the frequency sweep measurements were performed from 100 to $0.01 \mathrm{~Hz}$ within the linear range.

Recovery tests were performed in order to evaluate the structural recovery after the application of continuous shear conditions. They were composed of three consecutive segments, alternatively under small amplitude oscillatory conditions (at 1 $\mathrm{Hz}$ ) and in continuous shear at constant shear rate $\left(5 \mathrm{~s}^{-1}, 180 \mathrm{~s}\right)$. The steady values of G' and G' attained in the oscillatory segments before and after shear flow were compared.

All the experimental tests were done in duplicate or triplicate.

\section{Data treatment and modeling}

The experimental viscosity data can be correlated quite satisfactorily with the Carreau-Yasuda model: $^{32,33}$

$$
\eta=\eta_{\infty}+\frac{\eta_{0}-\eta_{\infty}}{\left[1+(a \dot{\gamma})^{b}\right]^{p}}
$$

where $\eta_{0}$ and $\eta_{\infty}$ represent the zero-shear rate viscosity and the infinite-shear rate viscosity, respectively. In this study, the high shear Newtonian pla- 
teau is beyond the experimental window and, hence, $\eta_{\infty}$ was arbitrarily set equal to $0.89 \mathrm{mPa}$ s, i.e. to the water viscosity at $25{ }^{\circ} \mathrm{C}$. The product $b p$ rules the slope of the flow curve in the power law region, while the critical shear rate $\gamma_{c}$ marking the onset of shear thinning is strictly related to the characteristic time $a$.

The mechanical spectra can be described with the generalized Maxwell model through the following expressions:

$$
G^{\prime}=\sum_{i=1}^{N} \frac{G_{i} \omega^{2} \lambda_{i}^{2}}{1+\omega^{2} \lambda_{i}^{2}} \quad G^{\prime \prime}=\sum_{i=1}^{N} \frac{G_{i} \omega \lambda_{i}}{1+\omega^{2} \lambda_{i}^{2}}
$$

where $G_{\mathrm{i}}$ and $\lambda_{\mathrm{i}}$ are the modulus and the relaxation time of the $i^{\text {th }}$ Maxwell element, respectively. In order to reduce the problem related to parameter correlation, a scaling criterion was adopted for the relaxation time sequence $\left(\lambda_{i+1}=10 \lambda_{i}\right)$ and not more than 5 Maxwell elements were taken into consideration for data fitting.

\section{Results and discussion}

The effects produced by amidation on the rheological behaviour of CMC solutions appeared to be more evident at low shear conditions and higher polymer concentrations. Comparing the flow curves of CMC and CMCAp solutions at equal concentration, the zero-shear-rate viscosity diminished after amidation and the Newtonian plateau became more extended towards higher shear conditions, as it can be observed in Fig. 1. Similar rheological effects of amidic moieties on carboxymethylcellulose have been observed by Barbucci et al. ${ }^{30}$

A satisfactory data correlation was provided by the Carreau-Yasuda equation. Accordingly, the above underlined effects can be concisely described

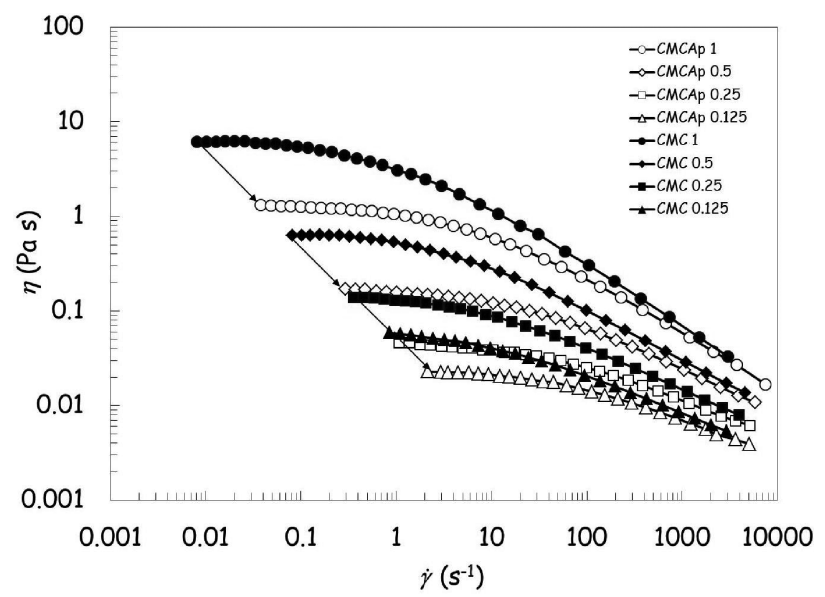

Fig. 1 - Steady viscosity curves of aqueous CMC and CMCAp solutions at different concentrations (expressed in $\% w / w)$

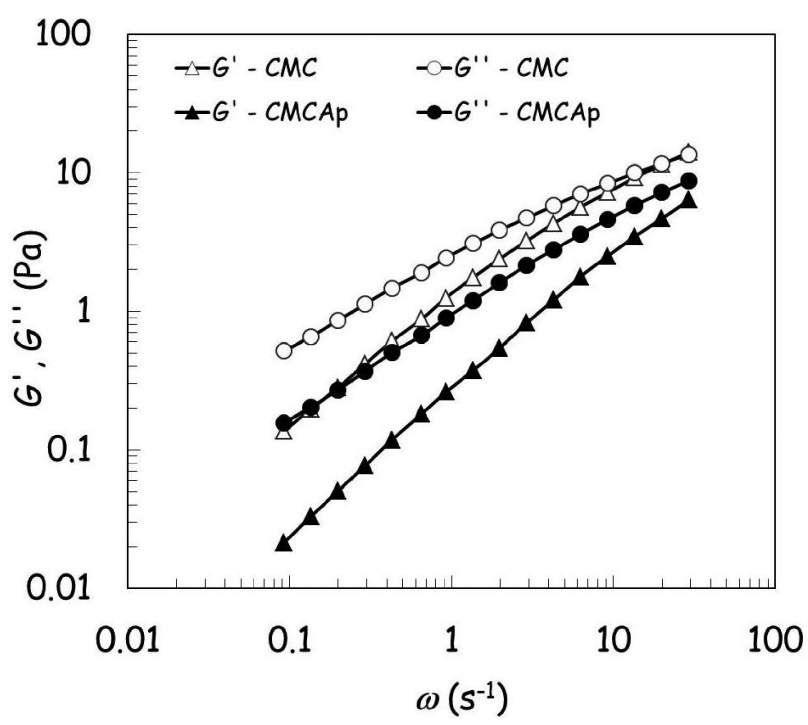

Fig. 2 - Mechanical spectra of aqueous CMC and CMCAp $1 \% \mathrm{w} / \mathrm{w}$ solutions

through the variations of two model parameters: the zero shear rate viscosity $\eta_{0}$ and the critical shear rate $\gamma_{\mathrm{c}}$.

The changes induced by amidation on the linear viscoelastic behaviour of solutions were coherent with those observed for their flow properties. At equal polymer concentration, the mechanical spectrum moved towards lower values of both moduli and the crossover point shifted to higher frequencies because of the faster relaxation modes. The example reported in Fig. 2 regards the $1 \%$ solutions. These effects can be evaluated more precisely by comparing the relaxation time spectra obtained from data correlation with the Maxwell generalized model, as illustrated in Fig. 3.

More drastic changes were produced by polymer crosslinking, particularly in linear viscoelastic conditions; this can be seen by comparing the re-

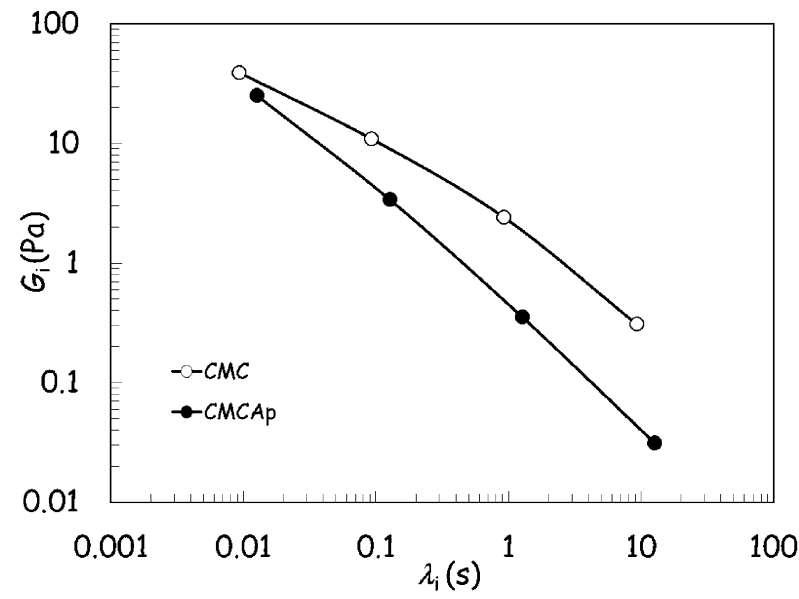

Fig. 3 - Relaxation time spectra of aqueous CMC and CMCAp $1 \% \mathrm{w} / \mathrm{w}$ solutions derived from data correlation with the Maxwell generalized model 

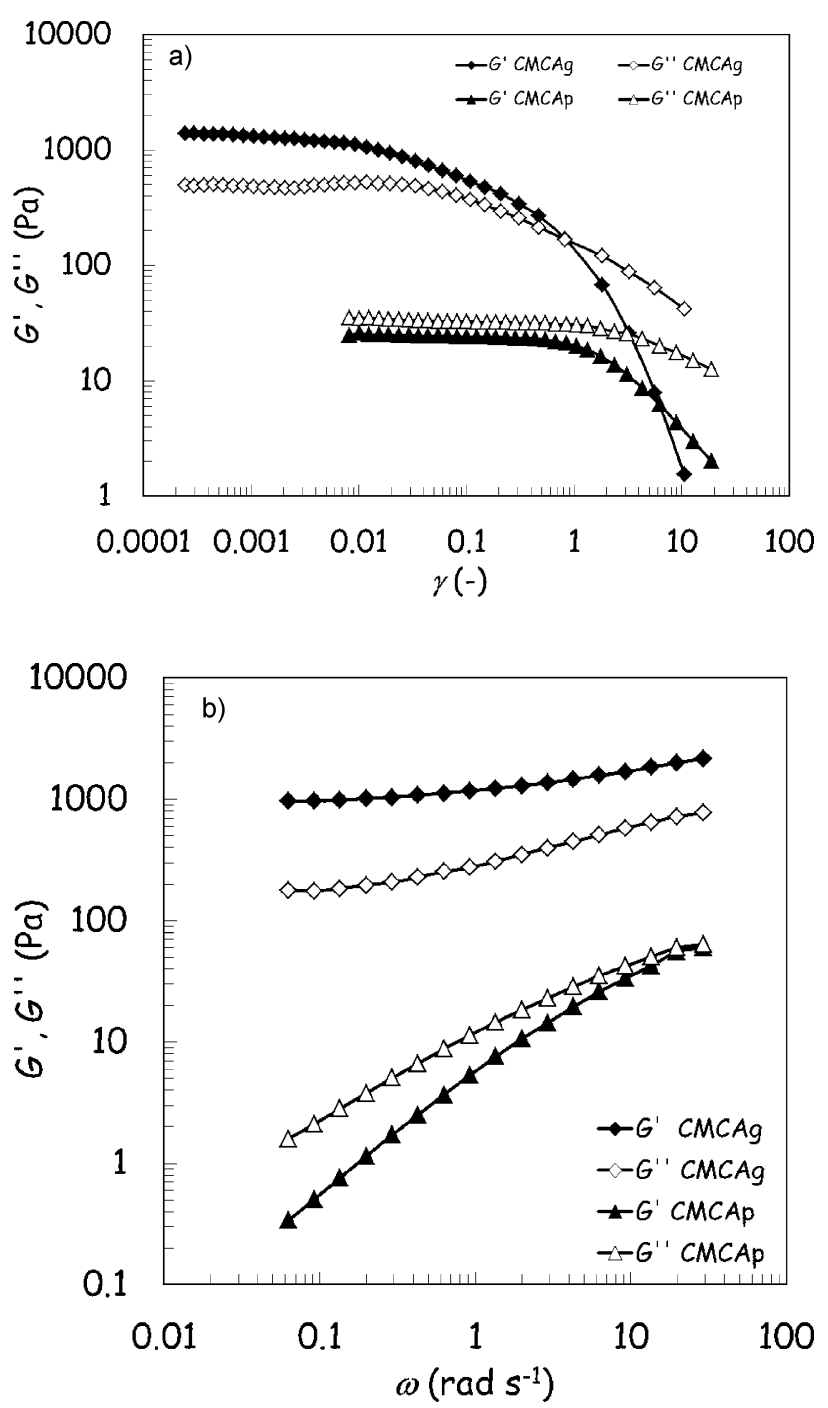

Fig. 4 - Viscoelastic responses of $2.5 \%$ CMCAp and $2.5 \%$ CMCAg systems; a) storage and loss moduli vs strain (from stress sweep test); b) mechanical spectra (from frequency sweep test)

sponses of both of CMCAp and CMCAg systems with the same concentration of soluble and crosslinked polymer $(2.5 \%$ for systems represented in Figs. $4 \mathrm{a}$ and $4 \mathrm{~b}$, respectively).

Stress sweep tests showed that both viscoelastic moduli of the CMCAg system were markedly higher, and in the nonlinear region, their profiles were different from those of the corresponding CMCAp solutions (Fig. 4a). For the latter systems, the transition from linear to nonlinear regime is described by monotonic decrease of both moduli. This behaviour is usually observed in ordinary polymeric solutions and labelled as type I according to the classification of LAOS responses..$^{34}$ Conversely, in the case of CMCAg systems, the storage modulus decreased monotonically with increasing strain whereas a weak overshoot appeared in the G" profile. Such a pattern (Type III) is typical of weakly structured fluids (physical gels or colloidal disper- sions). For CMCAg systems, the critical strain marking the borderline of the linear viscoelastic regime became sensibly lower (two orders of magnitude or more) than for CMCAp solutions.

The quite different profiles of the mechanical spectra of CMCAp and CMCAg systems reflect the neat changes produced by crosslinking in linear viscoelastic properties and relaxation modes, thus evidencing the structural transition from solution to physical gel (Fig. 4b). In the experimental window explored, the behaviour of the CMCAp solution was characterized by a prevailing viscous component $\left(G '>G^{\prime}\right)$ and short relaxation times, as it can be deduced by the crossover frequency. In the case of the crosslinked polymer, the storage modulus curve is placed clearly above G" values, and reaches a plateau condition at low frequencies, where the divergence from the linear viscoelastic responses of CMCAp is quite evident.

Studying the steady flow properties, we found that CMCAg systems exhibited much more pronounced shear thinning than CMCAp solutions, owing to their different structural conditions and shear-induced mechanisms. CMCAg systems behave as dispersions of gel microdomains, which may undergo significant deformations and spatial rearrangements with increasing shear rate.

Looking at the problems connected to the development of a novel polymeric fluid for viscosupplementation treatment, the first decisive step concerns its formulation in relation to the structural and rheological properties that the fluid must possess and exhibit. In particular, it should be easily injectable and display appropriate viscoelastic responses 'in situ' in the various kinematic conditions experienced by articular joints. Furthermore, an adequate resistance to enzymatic degradation and mechanical solicitations for prolonged times should be ensured. Blending CMCAp solutions and CMCAg microgel dispersions it is possible to finely adjust the composition ratio and the total polymer concentration in order to modulate the structural features and rheological properties of both systems.

The following figures illustrate how the rheological properties of CMCAp-CMCAg blends changed with increasing gel fraction at equal total polymer concentration. The steady viscosity strongly increased at low shear rates, while its change was almost negligible at high shear rates. As the gel fraction increased, the shear thinning character of the flow behaviour became more and more marked, being ruled by the level and extension of the Newtonian plateau (Fig. 5).

The experimental data can be correlated quite satisfactorily with the Carreau-Yasuda equation. The effects of increasing gel fraction $\phi_{\mathrm{g}}$ on viscous 


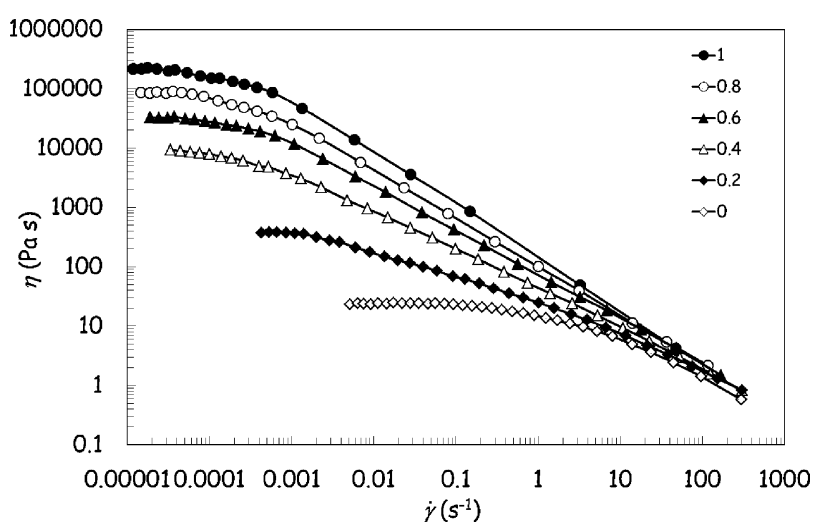

Fig. 5 - Steady viscosity curves of $2.5 \%$ CMCAp-CMCAg blends at different gel fractions (1 represents the polymer/gel ratio of 0/10; 0 represents a polymer/gel ratio of 10/0)

properties of CMCAp-CMCAg blends can be reasonably described through the only variations of the zero-shear rate viscosity $\eta_{0}$ and the critical shear rate $\gamma_{c}$, marking the border of the Newtonian plateau (Fig. 6). Thus, it can be noted that the most significant changes occurred in the $\phi_{\mathrm{g}}$ interval between 0.2 and 0.3 , where $\gamma_{c}$ drops significantly, and the rate of $\eta_{0}$ increase became slower. At higher $\phi_{\mathrm{g}}$ values, the increasing concentration of gel microdomains in the whole polymeric phase resulted only in an exponential $\eta_{0}$ increase with no appreciable effect on the critical shear rate.

Similarly, the linear viscoelastic behaviour of the blend significantly changed with increasing gel fraction. To clarify, the graphical representation reported in Fig. 7 involves only a partial subset of the mechanical spectra experimentally determined. As may be observed, the increasing gel fraction $\phi_{\mathrm{g}}$ led to an increase in the elastic character of the viscoelastic response, which was more pronounced at lower frequencies where the storage modulus increased more markedly than the viscous component. Consequently, a progressive change was ob-

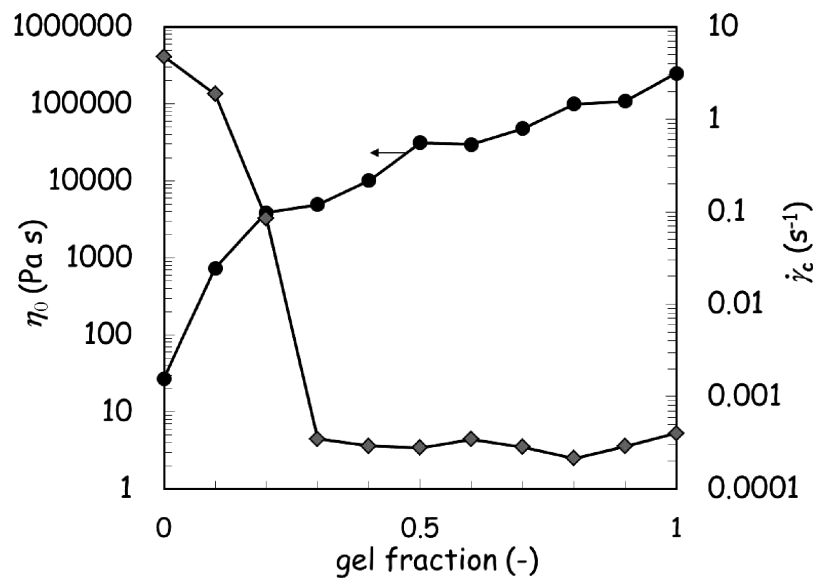

Fig. 6 - Zero-shear rate viscosity and critical shear rate vs gel fraction in $2.5 \%$ CMCAp-CMCAg blends

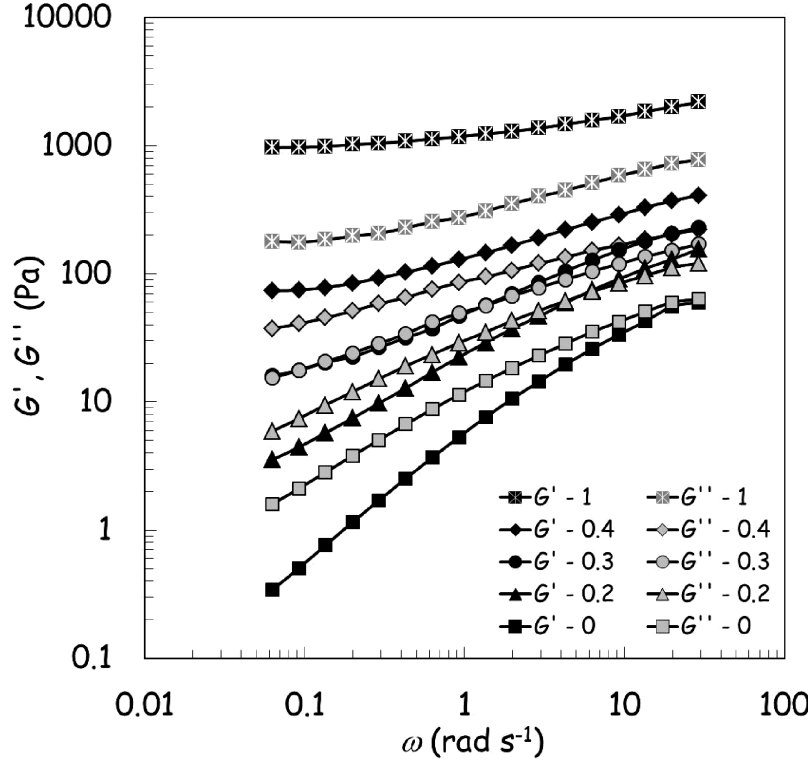

Fig. 7 - Mechanical spectra of $2.5 \%$ CMCAp-CMCAg blends at different gel fractions (0 represents the polymer/gel ratio of 10/0; 1 represents a polymer/gel ratio of 0/10)

served in the shape of the mechanical spectrum, passing from CMCAp solution to CMCAg system. According to the Winter-Chambon criterion ${ }^{35,36}$, the sol-gel transition could be located near $\phi_{g}=0.3$, since the traces of $G^{\prime}$ and $G$ " of system 7 were almost coincident and obey the same scaling law $\left(\mathrm{G}^{\prime} \cong \mathrm{G}^{\prime} \propto \omega^{\mathrm{n}}, \mathrm{n} \cong 0.45\right)$ in the experimentally explored frequency window.

The generalized Maxwell model provides a satisfactory data correlation and, consequently, the resulting relaxation time spectra can be profitably used for analysing the blending effects on the linear viscoelastic responses in the place of the mechanical spectra (Fig. 8). As the CMCAg content of the polymeric phase increased, the relative weight of the slowest relaxation modes became more import-

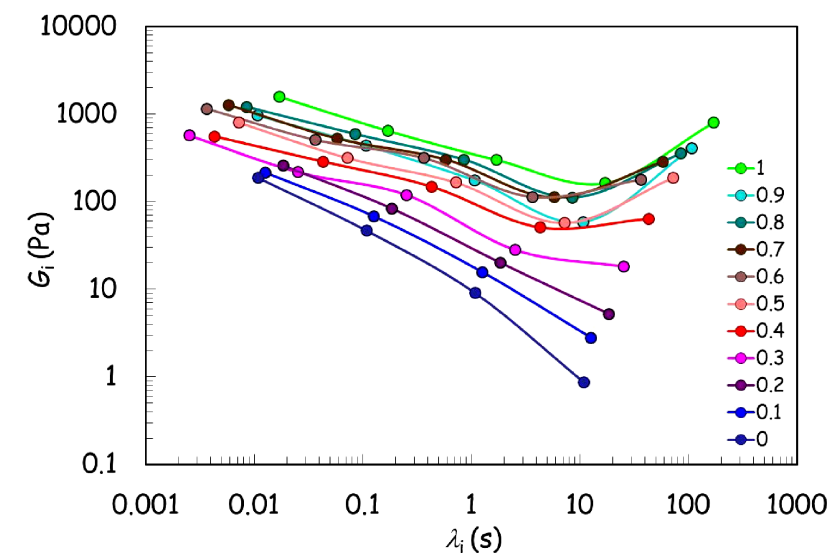

Fig. 8 - Relaxation time spectra derived from data correlation with the generalized Maxwell model for $2.5 \%$ CMCAp-CMCAg blends (1 represents the polymer/gel ratio of 0/10, 0 represents a polymer/gel ratio of 10/0) 
ant owing to the increasing contribution of gel microdomains towards a structural state close to jamming.

The analysis of the reversibility of linear viscoelastic responses (G', G”) can be particularly useful in view of the development of a novel viscosupplement, which must be injected, and hence undergoes short but intense shear conditions in the application phase. Recovery tests performed on CMCAp-CMCAg blends showed that the viscoelastic behaviour was completely reversible for CMCAp solution, since the values of G' and G" before and after shear flow were shown to be equal. On the other hand, only a partial recovery was attained for CMCAg system along the time segment following the application of a constant shear rate. The viscoelastic recovery decreased with increasing gel fraction.

Investigations were also carried out on the development of a pilot production process and evaluations were made on the potential effect of each processing step on the blend's final properties. Standard production implies a set of consecutive steps to obtain both components of the polymeric blend: $\mathrm{CMC}$ solubilization, $\mathrm{CMC}$ functionalization in order to obtain CMCA, purification of CMCA, concentration and freeze-drying of the polymer, crosslinking of the CMCA, purification of the obtained hydrogel, concentration and freeze-drying of the hydrogel. The final steps cover rehydration in saline solution, polymer and gel blending, filling in syringe and sterilization.

Among the several steps of the manufacturing process, some of them can strongly affect the elastoviscosity of the polymer blend. It has been observed that freeze drying, homogenization in saline solution and filling in syringes can produce a drastic decrease in loss and storage moduli in both soluble and crosslinked CMCA. Moreover, because viscosupplements are classified as pharmaceutical products or medical devices, an essential requirement for CMCA blends is represented by their capability to resist the sterilization process.

Several techniques based on chemical agents (EtO, plasma) or physical processes (heating, $\beta$ or $\gamma$ radiation) are widely used for sterilization in the pharmaceutical and medical devices industries. Among them, the most suitable and promising method in terms of efficacy and compatibility with solution or emulsions is represented by steam or moist heat. Conversely, it is known that heat induces degradation of CMC gels and polymer solutions ${ }^{37}$ and at high temperatures CMC degrades and permanently reduces in viscosity. Therefore, a rheological investigation has been dedicated to evaluate the potential degradation of CMCA blends during the

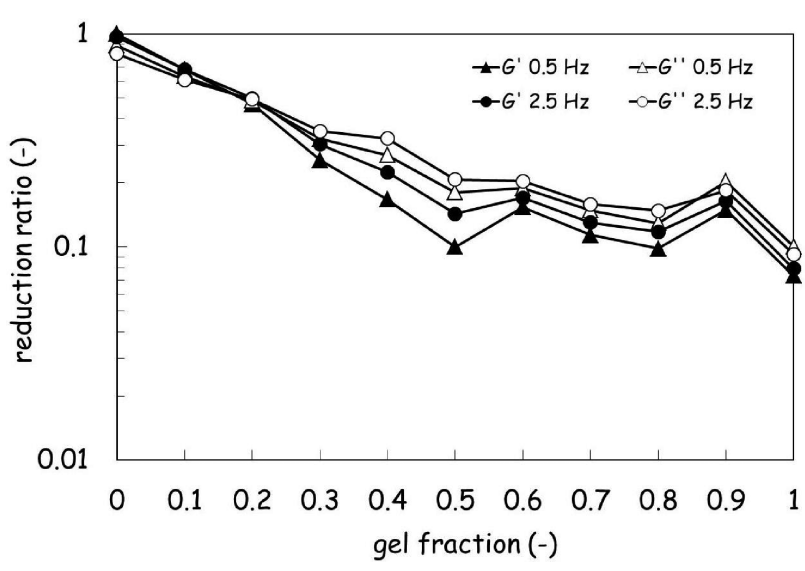

Fig. 9 - Reduction ratio of viscoelastic moduli between unsterile and sterile $2.5 \%$ CMCAp-CMCAg blends vs gel fraction at two reference frequencies $(0.5$ and $2.5 \mathrm{~Hz})$

manufacturing process, in particular that caused by steam sterilization.

Typical moist-heat sterilization cycles, based on exposition to temperature of $121^{\circ} \mathrm{C}$ for $15^{\prime}$ have been applied, and the mechanical spectra obtained for unsterile and sterile blends of equal total polymer concentration and gel fraction have been compared. As highlighted in Fig. 9, it can be seen that steam sterilization induced a loss of both elastic and viscous moduli due to the polymer degradation, and hence a reduction in the main properties of the device.

Consequently, the initial formulation must be readjusted in terms of total polymer concentration and gel fraction in order to compensate the negative effects produced by the manufacturing process on the rheological behaviour of the blend and to restore a correct balance among viscoelasticity, stability and easy injectability. In fact rheological properties of numerous viscosupplements available on the market cover a wide spectrum of values and sometimes they are quite different from those peculiar to healthy synovial fluids..$^{20,38-40}$

This suggests that the main requirements for such devices are represented by their viscoelastic properties in order to exhibit a proper response to the stress conditions within synovial joints, together with their capability to be easily injected through an $18-20 \mathrm{G}$ needle. It is also acknowledged that the values of the linear viscoelastic moduli measured at 0.5 and $2.5 \mathrm{~Hz}$ can represent a suitable way to evaluate how synovial fluid and viscosupplements react to walking and running conditions, respectively. ${ }^{20,41}$ Accordingly, they can serve to discriminate the behaviours of synovial fluids in normal and pathological conditions, as well as to assess the effects of viscosupplementation therapies. ${ }^{39,40,42}$ The experimental values obtained for a series of commercial viscosupplements are reported in Fig. 10a. The 

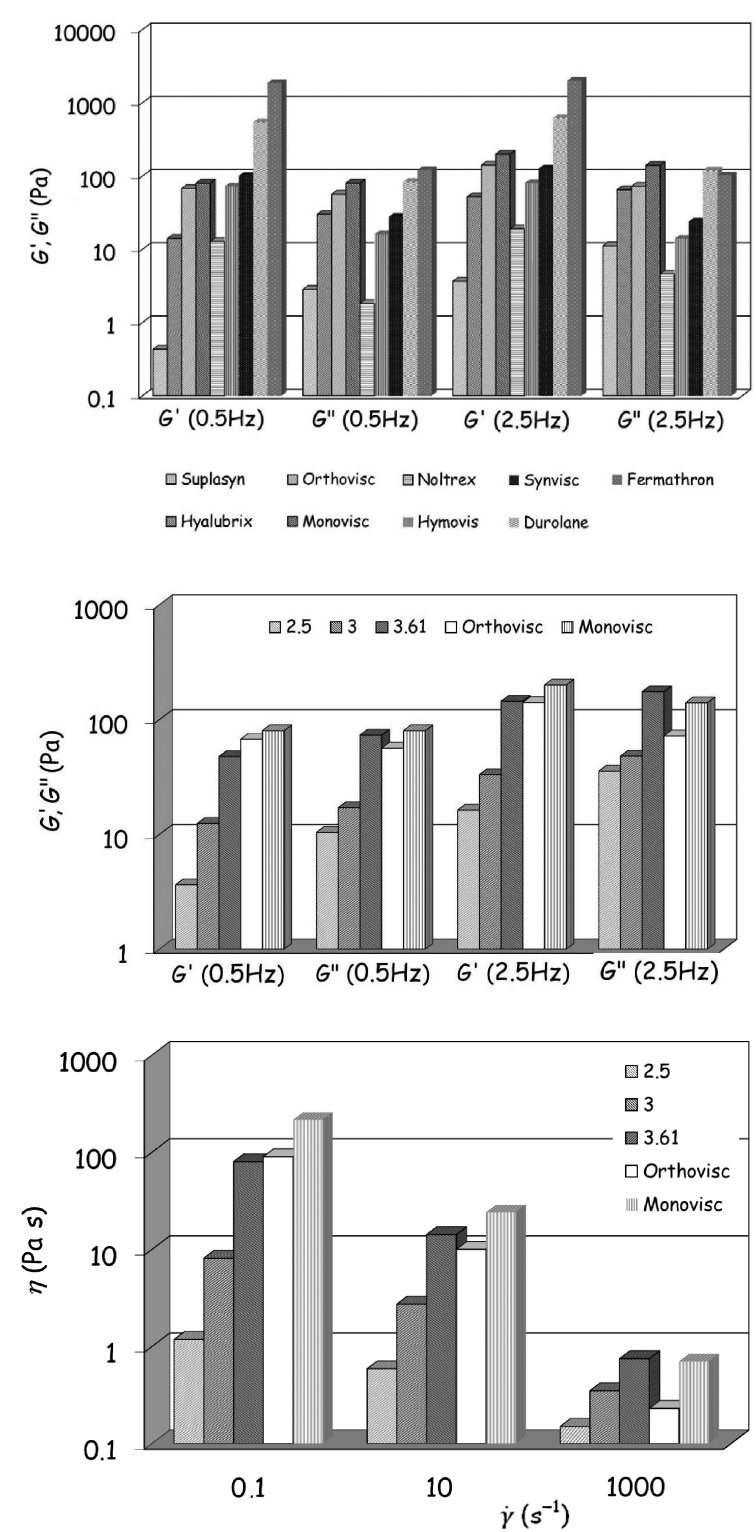

Fig. 10 - a: Viscoelastic moduli (at 0.5 and $2.5 \mathrm{~Hz}$ ) of the commercial viscosupplements listed below. $b$ : Viscoelastic moduli (at 0.5 and $2.5 \mathrm{~Hz}$ ) of the $6 / 4 \mathrm{CMCAp} / \mathrm{CMCAg}$ blends at $2.5 \%$, $3 \%$ and $3.61 \% \mathrm{w} / \mathrm{w}$ concentrations compared with those of two commercial products (Orthovisc and Monovisc). c: Viscosity values (at 0.1, 10 and $1000 \mathrm{~s}^{-1}$ ) of the 6/4 CMCAp/CMCAg blends at $2.5 \%, 3 \%$ and $3.61 \% \mathrm{w} / \mathrm{w}$ concentrations compared with those of two commercial products (Orthovisc and Monovisc). Commercial viscosupplement

Suplasyn (Mylan N.V., UK), non-heat degraded highly purified sodium hyaluronate $(500$ to $730 \mathrm{kDa}$ ), measured values, ref. 39.; Hyalubrix (Fidia farmaceutici S.p.A., Italy), sodium hyaluronate (1300 to $3600 \mathrm{kDa}$ ), from bacteria fermentation, estimated values, ref. 40.; Orthovisc (Anika Therapeutics MA, USA), highly purified sodium hyaluronate in physiologic saline, measured values, ref. 39.; Monovisc (Anika Therapeutics MA, USA) highly purified, partially crosslinked sodium hyaluronate in a phosphate buffered saline solution, estimated values, ref. 40.; Noltrex (JSC "Research center "Bioform", Russia), synthetic polyacrylamide hydrogel with silver ions, measured values in our laboratory; Hymovis (Fidia farmaceutici S.p.A., Italy), partially hydrophobized derivative of hyaluronic acid, estimated values, ref. 40.; Synvisc (Hylan G-F 20) (Genzyme Biosurgery, USA). blend 80/20 of Hylan A (high molecular weight hyaluronic acid) and Hylan B (crosslinked hyaluronic acid), measured values, ref. 39.: Durolane (Bioventus, USA), crosslinked hyaluronic acid, measured values in our laboratory.; Fermathron (Hyaltech Ltd. UK), sodium hyaluronate (1300 to $3600 \mathrm{kDa}$ ) in a phosphate buffered saline solution, from bacterial fermentation, measured values in our laboratory. quite large interval covered by both moduli at both frequencies is strictly related to the different mechanical spectra displayed by the systems considered, spanning from those typical of ordinary polymeric solutions to classical gel behaviours. Similar differences can also be found among novel systems proposed as candidates for viscosupplementation. ${ }^{40,42-45}$

Tests performed on blends with different gel fractions have shown that an acceptable structural recovery after continuous shear application (more than $80-90 \%)$ is attained when the gel fraction is lower than $50 \%$. Thus, the $6 / 4 \mathrm{CMCAp} / \mathrm{CMCAg}$ blend has been provisionally selected for the final investigation addressed to individuate the most suitable total polymer concentration, apt to compensate the loss in mechanical properties occurring during processing and sterilization. The viscoelastic moduli of three 6/4 CMCAp/CMCAg blends with different total polymer concentrations are compared with those of two commercial products in Fig. 10b, while Fig. 10c reports the viscosity values of the same systems at three different shear rates. From both figures, it can be inferred that the $3.61 \mathrm{w} / \mathrm{w}$ concentration provides adequate viscoelastic properties, which are combined with a proper injectability.

\section{Conclusions}

Blends with tailored viscoelastic properties can be obtained from CMCA in the form of polymer solutions and crosslinked hydrogels.

More specifically, the entire range between $10 / 0$ and $0 / 10$ of polymer/hydrogel ratio has been investigated, and the 5/5-6/4-7/3 polymer/hydrogel compositions seem to create a behaviour very close to the main characteristics of an HA-based product for viscosupplementation.

This study has demonstrated the importance of both polymer and hydrogel form in blends, because, if singularly used, (10/0 and $0 / 10$ of polymer/hydrogel samples), they are unable to preserve both storage and loss moduli.

Properties, such as lubrication and shock absorbing, together with an adequate resistance to processing and steam sterilization, can be assured by using a concentration of $3.61 \% \mathrm{w} / \mathrm{w}$.

The possibility to formulate CMCA in several polymer/hydrogel blends and in different concentrations, together with its biocompatibility and its slow enzymatic degradation, should be considered as a promising alternative to HA for the design of new products for viscosupplementation. 


\section{References}

1. Abramson, S. B., Attur, M., Arthritis Res. Ther. 11(3) (2009) 227. doi: http://dx.doi.org/10.1186/ar2655

2. Buckwalter, J. A., Mankin, H. J., J. Bone. Joint Surg. Am. 79 (1997) 600.

3. Buckwalter, J. A., Mankin, H. J., J. Bone. Joint Surg. Am. 79 (1997) 612.

4. Dawson, J., Linsell, L., Zondervan, K., Rose, P., Randall, T., Carr, A., Fitzpatrick, R., Rheumatology (Oxford) 43 (2004) 497. doi: http://dx.doi.org/10.1093/rheumatology/keh086

5. Day, J. S., Lau, E., Ong, K. L., Williams, G. R., Ramsey, M. L., Kurtz, S. M., J. Shoulder Elbow Surg. 19 (2010) 1115. doi: http://dx.doi.org/10.1016/j.jse.2010.02.009

6. Kwon, Y. W., Eisenberg, G., Zuckerman, J. D., J. Shoulder Elbow Surg. 22 (2013) 584. doi: http://dx.doi.org/10.1016/j.jse.2012.10.040

7. Litwic, A., Edwards, M. H., Dennison, E. M., Cooper C., Br. Med. Bull. 105 (2013) 185. doi: http://dx.doi.org/10.1093/bmb/lds038

8. Tanna, S., 2004. Background Papers of Priority Medicines for Europe and the World. Geneva, World Earth Organization.

9. Bitton, R., Am. J. Manag. Care 15 (8 Suppl) (2009) S230.

10. Dibonaventura, M. D., Gupta, S., McDonald, M., Sadosky, A., Pettitt, D., Silverman, S., Health Qual. Life Outcomes 10 (2012) 1 doi: http://dx.doi.org/10.1186/1477-7525-10-30

11. Gupta, S., Hawker, G. A., Laporte, A., Croxford, R., Coyte, $P$. C., Rheumatology (Oxford) 44 (2005) 1531. doi: http://dx.doi.org/10.1093/rheumatology/kei049

12. Jay, G. D., Torres, J. R., Warman, M. L., Laderer, M. C., Breuer, K. S., Proc. Natl. Acad. Sci. USA 104 (2007) 6194. doi: http://dx.doi.org/10.1073/pnas.0608558104

13. Purbach, B., Hills, B. A., Wroblewski, B. M., Clin. Orthop. Relat. Res. 396 (2002) 115. doi: http://dx.doi.org/10.1097/00003086-200203000-00020

14. Hills, B. A., Crawford, R. W., J. Arthroplasty 18 (2003) 499. doi: http://dx.doi.org/10.1016/S0883-5403(03)00072-X

15. McNary, S. M., Athanasiou, K. A., Reddi, A. H., Tissue Eng, Part B Rev. 18 (2012) 88. doi: http://dx.doi.org/10.1089/ten.teb.2011.0394

16. Schmidt, T. A., Gastelum, N. S., Nguyen, Q. T., Schumacher, B. L., Sah, R. L., Arthritis Rheum. 56 (2007) 882. doi: http://dx.doi.org/10.1002/art.22446

17. Crockett, R., Tribol. Lett. 35 (2009) 77. doi: http://dx.doi.org/10.1007/s11249-009-9430-x

18. Nitzan, D. W., Nitzan, U., Dan, P., Yedgar, S., Rheumatology (Oxford) 40 (2001) 336. doi: http://dx.doi.org/10.1093/rheumatology/40.3.336

19. Dahl, L. B., Dahl, I. M., Engstrom-Laurent, A., Granath, K., Ann. Rheum. Dis. 44 (1985) 817. doi: http://dx.doi.org/10.1136/ard.44.12.817

20. a) Balazs, E. A., Surg. Technol. Int., 12 (2004) 278; b) Balazs, E. A., Denlinger, J. L., J. Rheumatol. Suppl. 39 (1993) 3.

21. Stitik, T. P., Levy, J. A., Am. J. Phys. Med. Rehabil. 85 (Suppl 11) (2006) S32. doi: http://dx.doi.org/10.1097/01.phm.0000245677.20294.c2
22. Zhang, W., Nuki, G., Moskowitz, R. W., Abramson, S., Altman, R. D., Arden, N. K., Bierma-Zeinstra, S., Brandt, K. D., Croft, P., Doherty, M., Dougados, M., Hochberg, M., Hunter, D. J., Kwoh, K., Lohmander, L. S., Tugwell, P., Osteoarthritis Cartilage 18 (2010) 476. doi: http://dx.doi.org/10.1016/j.joca.2010.01.013

23. Gigante, A., Callegari, L., Rheumatol. Int. 31 (2011) 427. doi: http://dx.doi.org/10.1007/s00296-010-1660-6

24. Ogston, A. G., Stanier, J. E., J. Physiol. 119 (1953) 244. doi: http://dx.doi.org/10.1113/jphysiol.1953.sp004842

25. Pozo, M. A., Balazs, E. A., Belmonte, C., Exp. Brain. Res. 116 (1997) 3. doi: http://dx.doi.org/10.1007/PL00005742

26. Moreland, L. W., Arthritis Res. Ther. 5 (2003) 54. doi: http://dx.doi.org/10.1186/ar623

27. Balazs, E. A., Struct. Chem. 20 (2009) 341. doi: http://dx.doi.org/10.1007/s11224-009-9435-y

28. Martens, P. B., Arthritis Rheum. 44 (2001) 978. doi: http://dx.doi.org/10.1002/1529-0131(200104)44:4<978 ::AID-ANR156>3.0.CO;2-N

29. Puttick, M. P., Wade, J. P., Chalmers, A., Connell, D. G., Rangno, K. K., J. Rheumatol. 22 (1995) 1311.

30. Barbucci, R., Leone, G., Monici, M., Pantalone, D., Fini, M., Giardino, R., J. Mater. Chem. 15 (2005) 2234. doi: http://dx.doi.org/10.1039/b503399c

31. Leone, G., Delfini, M., Di Cocco, M. E., Borioni, A., Barbucci, R., Carbohyd. Res. 343 (2008) 317. doi: http://dx.doi.org/10.1016/j.carres.2007.10.017

32. Carreau, P. J., Ph.D. Thesis, University of Wisconsin, Madison, 1968.

33. Yasuda, K., PhD Thesis, Massachusetts Institute of Technology, Cambridge, 1979.

34. Hyun, K., Kim, S. H., Ahn, K. H., Lee, S. J., J. Non-Newtonian Fluid Mech. 107 (2002) 51.

35. Winter, H. H., Chambon, F., J. Rheol. 30 (1986) 367. doi: http://dx.doi.org/10.1122/1.549853

36. Chambon, F., Winter, H. H., J. Rheol. 31 (1987) 683. doi: http://dx.doi.org/10.1122/1.549955

37. Chu, P. I., Doyle, D., Pharm. Dev. Technol. 4 (1999) 553. doi: http://dx.doi.org/10.1081/PDT-100101394

38. Fam, H., Bryant, J. T., Kontopoulou, M., Biorheology, 2007, 44, 59-74.

39. Bhuanantanondh, P., M.A.Sc. Thesis, University of British Columbia, 2009.

40. Finelli, I., Chiessi, E., Galesso, D., Renier, D., Paradossi, G., Biorheology 48 (2011) 263.

41. Balasz, E. A., Univ. Mich. Med. Center J. 9 (1968) Special Issue 255.

42. Borzacchiello, A., Mayol, L., Schiavinato, A., Ambrosio, L., J. Biomed. Mat. Res. A 92 (2010) 1162.

43. Pelletier, S. Hubert, P., Payan, E., Marchal, P. Choplin, L., Dellacherie, E., J. Biomed. Mat. Res. 54 (2001) 102. doi: http://dx.doi.org/10.1002/1097-4636(200101)54:1<102 ::AID-JBM12>3.0.CO;2-1

44. Casuso, P., Pérez-San Vicente, A., Iribar, H., Gutiérrez-Rivera, A., Izeta, A., Loinaz, I., Cabañero, G., Grande, H. J., Odriozola, I., Dupin, D., Chem. Comm. 50 (2014) 15199. doi: http://dx.doi.org/10.1039/C4CC05735J

45. Mayol, L., Biondi, M., Russo, L., Malle, B. M., Schwach-Abdellaouid, K., Borzacchiello, A., Carbohyd. Polym. 102 (2014) 110. doi: http://dx.doi.org/10.1016/j.carbpol.2013.11.003 